\title{
First principles simulation of light propagation and exciton dynamics in metal cluster nanostructures
}

\author{
Polina G. Lisinetskaya, Merle I. S. Röhr, and Roland Mitrić \\ Institut für Physikalische und Theoretische Chemie, \\ Universität Würzburg, D-97074 Würzburg, \\ Germany. E-mail: roland.mitric@uni-wuerzburg.de
}

\begin{abstract}
We present a theoretical approach for the simulation of the electric field and exciton propagation in ordered arrays constructed of molecular-sized noble metal clusters bound to organic polymer templates. In order to describe the electronic coupling between individual constituents of the nanostructure we use the ab initio parameterized transition charge method which is more accurate than the usual dipole-dipole coupling. The electronic population dynamics in the nanostructure under an external laser pulse excitation is simulated by numerical integration of the time-dependent Schrödinger equation employing the fully coupled Hamiltonian. The solution of the TDSE gives rise to time-dependent partial point charges for each subunit of the nanostructure, and the spatiotemporal electric field distribution is evaluated by means of classical electrodynamics methods. The time-dependent partial charges are determined based on the stationary partial and transition charges obtained in the framework of the TDDFT. In order to treat large plasmonic nanostructures constructed of many constituents, the approximate self-consistent iterative approach presented in [Lisinetskaya and Mitrić, Phys. Rev. B 035433, 2014] is modified to include the transition-chargebased interaction. The developed methods are used to study the optical response and exciton dynamics of $\mathrm{Ag}_{3}^{+}$and porphyrin- $\mathrm{Ag}_{4}$ dimers. Subsequently, the spatio-temporal electric field distribution in a ring constructed of ten porphyrin- $\mathrm{Ag}_{4}$ subunits under the action of circularly-polarized laser pulse is simulated. The presented methodology provides a theoretical basis for the investigation of coupled light-exciton propagation in nanoarchitectures built from molecular size metal nanoclusters in which quantum confinement effects are important.
\end{abstract}




\section{INTRODUCTION}

Noble metal nanoclusters are nowadays widely recognized as promising building blocks for novel electro-optical devices [1-9]. Their unique optical, chemical and electronic properties find applications in nanoscience and nanotechnology, providing nonlinear optical sources $[5,10,11]$, biosensors [12-15], energy transport systems [16-18], solar cell elements [19, 20], etc. Of particular interest for nanoelectronics and plasmonics are assemblies of nanoparticles, which demonstrate novel functionalities exceeding those of individual particles [21].

Theoretical simulations play an important role in developing ultrasmall optical and electronic devices not only by explaining the mechanisms underlying experimentally observed processes, but also by proposing and examining new systems with interesting properties and new directions for the experimental research. One of the exciting opportunities opened by ultra-small metal cluster aggregates is the ability to transport electronic excitation and to control its localization in space and time. For aggregates constructed of 10-100 nm sized nanoparticles it was both theoretically and experimentally demonstrated, that ultrafast spatial energy localization can be achieved and controlled by phase-shaped external laser pulses [22-24]. Along with that, the possibility to control light propagation in structured arrays of nanoparticles was studied by theoretical and experimental means [25-28]. To describe light interactions with nanoparticles and their aggregates in this size range, various theoretical approaches have been employed including the discrete-dipole approximation (DDA) [29], the extended Mie theory [16, 30-32] and the finite-difference time-domain (FDTD) method $[25,33,34]$.

Pushing the frontier towards further reduction of the size of plasmonic devices, one eventually reaches the size regime where "each atom counts", i.e. alteration of the number of atoms in a cluster or even their spatial configuration leads to dramatic changes of their optical and chemical properties [3, 5, 35-39]. In order to describe the interaction of these ultra-small clusters with electric fields, the well-developed theoretical approaches mentioned above were coupled to quantum chemistry to account for the intrinsically quantum nature of the clusters [40-43]. At the moment the attention of researchers is mainly devoted to model few-energy-level systems with degenerate electronic excited states, while for the development of novel plasmonic systems and for the interpretation of experimental results the realistic description of the electronic structure of a single constituent as well as of the aggregate as a 
whole is mandatory. Additionally, due to the small size of individual clusters it is essential to take into account the effects of the substrate on which cluster arrays are deposited when modeling the electronic and optical properties of the nanostructures.

The problem of the simulation of light propagation in noble metal cluster arrays with a realistic description of single-cluster electronic structure has been addressed in our previous work [44]. The proposed approach included the construction of an array excitonic Hamiltonian based on ab initio quantum chemical calculations, solving time-dependent Schrödinger equation (TDSE) to simulate the optical response of the array, and finally using the obtained quantum-mechanical time-dependent dipole moments to obtain the electric field distribution by means of classical electrodynamics. Additionally, a self-consistent iterative method of solving the TDSE for arrays of well-separated noble metal clusters was proposed. The iterative method allows for treating large arrays consisting of many subunits with tens of electronic states per subunit included, which is mandatory even for small metal clusters. Recently, using this method, the possibility of optimal control of light propagation in a T-shaped structure constructed of $\mathrm{Ag}_{8}$ clusters has been also demonstrated [45]. The shortcoming of the described method is that it is based on the dipole approximation to describe the interaction between different clusters and thus can not be applied to arrays built up of subunits that are larger than their spatial separation.

In the field of energy transfer in biological systems several approaches to evaluate the interaction between large closely-placed molecules have been proposed, which go beyond the dipole approximation. Among them are the transition density cube (TDC) [46] and transition charge (TC) [47] methods. Within the TDC approach the transition density for each subunit is calculated on a grid using first principles methods such as TDDFT, and the interaction between subunits is subsequently evaluated as the Coulomb interaction between two charge densities. The TC approach is an approximation of the TDC method on fitting of the electrostatic potential produced by the transition density with a set of point charges located at nuclear positions. The interaction between two sets of these charges representing two molecules is then calculated according to the Coulomb law. The TC method is computationally very efficient and has the advantage that the TCs once calculated for a selected molecule can be further used to estimate the intermolecular interactions at different mutual positions and orientations of molecules.

In the current contribution we combine our approach for the simulation of light propa- 
gation in small noble-metal cluster arrays with the TC method for the description of the cluster-cluster interaction beyond the dipole approximation. The developed method is applied to study the optical response and light propagation in porphyrin- $\mathrm{Ag}_{4}\left(\mathrm{PorphAg}_{4}\right)$ oligomers. The PorphAg 4 subunit is chosen due to the following reasons: First, the porphyrin molecules are well known for their property to strongly bind metal atoms to the central site, and quantum chemical simulations reveal that small metal clusters are strongly bound to the central site strongly as well [48]. Secondly, modern chemical methods allow for synthesis of a large variety of sophisticated one-, two-, and three-dimensional structures with the porphyrin molecule serving as a building block [49, 50]. Therefore the porphyrin oligomers seem to be a promising support for the spatial organization of ultra-small noblemetal clusters. As an example of a two-dimensional PorphAg ${ }_{4}$ array, in the current work we consider a plane ring constructed of ten PorphAg 4 subunits and study the response of the ring to the irradiation of circularly polarized light. The simulations reveal that this system is sensitive to the direction of the polarization plane rotation.

\section{THEORY}

\section{A. Excitonic Hamiltonian for the cluster array}

Consider an array consisting of $N$ building blocks to which we will further refer as to "clusters". The electronic Hamiltonian of the array irradiated by an external laser field can be constructed based on the single-cluster Hamiltonians $\mathrm{H}_{I}^{0}$, the pairwise cluster interaction operator $\mathrm{V}_{I J}$, and the interaction with electric field $\mathrm{V}_{I}^{e x t}$ in the following way:

$$
\mathrm{H}=\sum_{I=1}^{N} \mathrm{H}_{I}^{0} \otimes \mathrm{I}_{I}+\sum_{I=1}^{N} \sum_{J>I} \mathrm{~V}_{I J} \otimes \mathrm{I}_{I J}+\sum_{I=1}^{N} \mathrm{~V}_{I}^{e x t} \otimes \mathrm{I}_{I}=\mathrm{H}_{\text {arr }}+\sum_{I=1}^{N} \mathrm{~V}_{I}^{e x t} \otimes \mathrm{I}_{I} .
$$

Here, $\mathrm{I}_{I}$ and $\mathrm{I}_{I J}$ are the identity operators acting on the electrons of all clusters in the array, except the cluster $I$ or $I$ and $J$, respectively.

We consider a nanostructure built up of individual components, which are much smaller than the wavelength of the external laser field. Under this assumption each single cluster can be represented by a dipole emitter and the interaction with the external field can be described by a dipole term $\mathrm{V}_{I}^{e x t} \approx-\boldsymbol{\mu}_{I} \cdot \varepsilon_{0}(t)\left(e^{i\left(\mathbf{k}_{\omega} \cdot \mathbf{R}_{I}-\omega t\right)}+c . c\right)$. Here $\boldsymbol{\mu}_{I}$ is the dipole moment operator of the $I$-th cluster located at the cluster charge center $\mathbf{R}_{I} ; \boldsymbol{\varepsilon}_{0}(t), \mathbf{k}_{\omega}$ and 
$\omega$ represent the time envelope, wave vector and angular frequency of the electric field. In this manner the spatial variation of the external electric field over the whole array is taken into account by the position-dependent phase.

Interaction between clusters within the described approach is considered to be purely electromagnetic. Since the distance between neighboring array subunits is comparable to the size of a subunit, the dipole approximation cannot be used to simulate the clustercluster interaction and the spatial distribution of electrons and nuclei of a single cluster has to be taken into account. For this purpose, we employ the electrostatic-potential-derived transition charges [47], which allow for describing the interaction between molecules that are large compared to the distance between them. These charges are obtained by fitting the electrostatic potential produced by electrons and nuclei of a molecule with a set of point charges located at the positions of the nuclei:

$$
\varphi_{i j}(\mathbf{r})=-\int \frac{\rho_{i j}\left(\mathbf{r}^{\prime}\right)}{\left|\mathbf{r}-\mathbf{r}^{\prime}\right|} \mathrm{d} \mathbf{r}^{\prime}+\sum_{a} \frac{Q_{a}}{\left|\mathbf{r}-\mathbf{r}_{a}\right|} \approx \sum_{a} \frac{q_{i j}^{a}}{\left|\mathbf{r}-\mathbf{r}_{a}\right|}
$$

For $i \neq j$ the transition charges are calculated based on the transition density $\rho_{i j}$ between electronic states $i$ and $j$, while for $i=j$ the density $\rho_{i i}$ corresponds to the one-electron density and the contribution of nuclear charges $Q_{a}$ is taken into account. Thus the transition charges $q_{i i}^{a}$ are the same as the partial atomic charges derived based on the electrostatic potential fit for the electronic state $i$.

In this approximation the interaction operator $\mathrm{V}_{I J}$ is written in the Coulomb form:

$$
\mathrm{V}_{I J}=\sum_{a b} \frac{\mathrm{q}_{I, a} \mathrm{q}_{J, b}}{\left|\mathbf{r}_{I, a}-\mathbf{r}_{J, b}\right|}
$$

Here $\mathbf{r}_{I, a}$ and $\mathbf{r}_{J, b}$ stand for positions of nucleus $a$ in cluster $I$ and nucleus $b$ in cluster $J$, respectively; the summation goes over all nuclei to which the transition charges are assigned. The operator $\mathrm{q}_{I, a}$ is defined in the following way:

$$
\mathrm{q}_{I, a}=\sum_{i j} q_{i j}^{I, a}\left|\Psi_{i}^{I}\right\rangle\left\langle\Psi_{j}^{I}\right|
$$

where $\left|\Psi_{i}^{I}\right\rangle$ is the eigenfunction of the $I$ th cluster Hamiltonian $\mathrm{H}_{I}^{0}$ corresponding to the electronic state energy $E_{i}^{I}$, and $q_{i j}^{I, a}$ stands for the transition charge of the $I$ th cluster located at the position of nucleus $a$, derived based on the transition density between electronic states $i$ and $j$. 
Considering Eq. (1) we see that the first two terms are time-independent and are completely determined by the array structure and the type of individual constituents. Therefore the natural basis for solving the TDSE with Hamiltonian (1) is the basis spanned by the eigenfunctions of the time-independent part of the Hamiltonian $\left(\mathrm{H}_{\text {arr }}\right)$.

\section{B. Eigenfunctions and eigenenergies of $\mathrm{H}_{\text {arr }}$}

We represent the coupled array Hamiltonian $\mathrm{H}_{\text {arr }}$ using the tensor product basis constructed from the eigenfunctions of the individual clusters:

$$
\left|\phi_{i j \ldots z}\right\rangle=\left|\Psi_{i}^{1}\right\rangle \otimes\left|\Psi_{j}^{2}\right\rangle \otimes \ldots \otimes\left|\Psi_{z}^{N}\right\rangle
$$

where the indices $i, j, \ldots, z$ run over all included electronic states for each individual cluster, and superscripts $1,2, \ldots, N$ denote the index number of the cluster in the array. Since the Hamiltonian $\mathrm{H}_{\text {arr }}$ contains only one-cluster $\left(\mathrm{H}_{I}^{0}\right)$ and two-cluster $\left(\mathrm{V}_{I J}\right)$ terms, all matrix elements with three or more different indices in the set $i j \ldots z$ are zero due to orthogonality of the single-cluster eigenfunctions $\left\langle\Psi_{i^{\prime}}^{I} \mid \Psi_{i}^{I}\right\rangle=\delta_{i i^{\prime}}$. The diagonal matrix elements have the following form:

$$
\left(\mathrm{H}_{a r r}\right)_{i j \ldots z, i j \ldots z}=\sum_{I=1}^{N} E_{k_{I}}^{I}+\sum_{\substack{I=1 \\ J>I}}^{N} \sum_{a b} \frac{q_{k_{I} k_{I}}^{I, a} q_{k_{J} k_{J}}^{J, b}}{\left|\mathbf{r}_{I, a}-\mathbf{r}_{J, b}\right|},
$$

where $k_{I}$ is the the index of the electronic state of the $I$-th cluster in the set $i j \ldots z$. The non-diagonal elements of the matrix $\left(\mathrm{H}_{\text {arr }}\right)$ with only one different index are:

$$
\left(\mathrm{H}_{a r r}\right)_{i j \ldots k_{I} \ldots z, i j \ldots k_{I}^{\prime} \ldots z}=\sum_{\substack{J=1 \\ J \neq I}}^{N} \sum_{a b} \frac{q_{k_{I} k_{I}^{\prime}}^{I, a} q_{k_{J} k_{J}}^{J, b}}{\left|\mathbf{r}_{I, a}-\mathbf{r}_{J, b}\right|} .
$$

Finally, the elements of the matrix $\left(\mathrm{H}_{\text {arr }}\right)$ with two different indices read:

$$
\left(\mathrm{H}_{a r r}\right)_{i j \ldots k_{I} \ldots k_{J} \ldots z, i j \ldots k_{I}^{\prime} \ldots k_{J}^{\prime} \ldots z}=\sum_{a b} \frac{q_{k_{I} k_{I}^{\prime}}^{I, a} q_{k_{J} k_{J}^{\prime}}^{J, b}}{\left|\mathbf{r}_{I, a}-\mathbf{r}_{J, b}\right|} .
$$

After the matrix has been constructed, the eigenvalue problem is solved

$$
\mathrm{H}_{\text {arr }}\left|\psi_{p}\right\rangle=E_{p}\left|\psi_{p}\right\rangle
$$


which yields the set of the array excitonic states with eigenenergies $E_{p}$ and eigenvectors $\left|\psi_{p}\right\rangle$ represented in the basis (5) by the set of coefficients $C_{i j \ldots z}^{p}$ :

$$
\left|\psi_{p}\right\rangle=\sum_{i j \ldots z} C_{i j \ldots z}^{p}\left|\phi_{i j \ldots z}\right\rangle
$$

\section{Construction of $\mathrm{H}_{\text {arr }}$ based on TDDFT}

Eqs. (6)-(8) indicate that the essential quantities needed for construction of the array Hamiltonian are the electronic state energies of each single constituent and the transition charges between all electronic states included in the simulations. In general, for molecularsized clusters, these quantities can be obtained using any ab initio or semiempirical electronic structure method. In the current work we use the linear response time-dependent density functional theory (TDDFT) in order to obtain the energies of the electronic states since this method is efficient and can be applied to relatively large complex systems. To determine the transition charges, the corresponding transition densities are required. Notice that the full set of transition densities including the transitions among excited states cannot be calculated using standard linear responseTDDFT routines and therefore an approximate procedure presented in detail in Ref. [51] will be used here.

Briefly, the excited state electronic wavefunction is approximated by the configuration interaction singles-like (CIS) expansion:

$$
\left|\Psi_{k}(\mathbf{r})\right\rangle=\sum_{i, a} c_{i, a}^{k}\left|\Phi_{i, a}^{C S F}(\mathbf{r})\right\rangle
$$

where $\left|\Phi_{i, a}^{C S F}(\mathbf{r})\right\rangle$ is a singlet spin-adapted configuration state function (CSF) defined as:

$$
\left|\Phi_{i, a}^{C S F}(\mathbf{r})\right\rangle=\frac{1}{\sqrt{2}}\left(\left|\Phi_{i \alpha}^{a \beta}(\mathbf{r})\right\rangle+\left|\Phi_{i \beta}^{a \alpha}(\mathbf{r})\right\rangle\right)
$$

and $\left|\Phi_{i \alpha}^{a \beta}(\mathbf{r})\right\rangle\left(\left|\Phi_{i \beta}^{a \alpha}(\mathbf{r})\right\rangle\right)$ is a Slater determinant with two unpaired electrons, one on the occupied Kohn-Sham (KS) orbital $i$ with spin $\alpha(\beta)$ and another on the virtual orbital $a$ with spin $\beta(\alpha)$. The expansion coefficients $c_{i, a}^{k}$ in Eq. (11) are determined on physical grounds by requiring that the wavefunction in Eq. (11) leads to the same density response as the one obtained by the linear response TDDFT procedure. Thus, for non-hybrid functionals without exact exchange the coefficients $c_{i, a}^{k}$ are given by: 


$$
c_{i, a}^{k}=\left(\frac{\epsilon_{a}-\epsilon_{i}}{\omega_{k}}\right)^{-1 / 2}\left(X_{i a}^{k}+Y_{i a}^{k}\right),
$$

where $\epsilon_{i}$ and $\epsilon_{a}$ are the Kohn-Sham orbital energies of $i$-th occupied and $a$-th virtual single electron orbitals, respectively, $\omega_{k}$ is the excitation energy of the $k$-th excited state, and $X^{k}$ and $Y^{k}$ represent the solution of the TDDFT eigenvalue problem $[52,53]$. This allows one to define the set of mutually orthogonal electronic wavefunctions $\left|\Psi_{k}(\mathbf{r})\right\rangle$ which can be used as an approximate basis to represent the electronic eigenstates of a cluster.

The transition density in Eq. (2) is defined as

$$
\rho_{i j}\left(\mathbf{r}_{1}\right)=N_{e l} \int \ldots \int \Psi_{i}^{*}(\mathbf{x}) \Psi_{j}(\mathbf{x}) \mathrm{d} \mathbf{x}_{2} \ldots \mathrm{d} \mathbf{x}_{N_{e l}} \mathrm{~d} \sigma_{1},
$$

where $\mathbf{x}$ stands for spatial and spin coordinates of all electrons in the cluster, $N_{e l}$ is the number of electrons, the integration runs over all spatial and spin coordinates of all electrons except the spatial coordinates of the first one.

Consequently, Eqs. (11) and (12) are substituted into Eq. (14) and the resulting integral is calculated taking into account mutual orthogonality of the KS orbitals. Finally, the expressions for single-cluster transition densities between all the states of interest are obtained

$$
\begin{gathered}
\rho_{00}=2 \sum_{a} \psi_{a}^{*} \psi_{a}, \\
\rho_{0 i}=2 \sum_{a r} c_{a, r}^{i} \psi_{a}^{*} \psi_{r}, \\
\rho_{i j}=2\left(\delta_{i j} \rho_{00}+\sum_{a r s} c_{a, r}^{i *} c_{a, s}^{j} \psi_{r}^{*} \psi_{s}-\sum_{a b r} c_{a, r}^{i *} c_{b, r}^{j} \psi_{a}^{*} \psi_{b}\right),
\end{gathered}
$$

where $\{\psi\}$ are the KS orbitals of the cluster, indices $a, b$ run over all occupied and $r, s$ over all virtual orbitals. After the transition densities have been calculated, the corresponding transition charges can be straightforwardly determined via Eq. (2).

\section{Optical response to the external laser field}

Once the excitonic Hamiltonian is constructed, the electron dynamics in the cluster array induced by an external electromagnetic field can be simulated fully quantum-mechanically 
using the method described in Ref. [44]. Briefly, the total time-dependent wavefunction of the whole cluster array is expanded in the basis spanned by the excitonic eigenfunctions $\left|\psi_{p}\right\rangle$ (see Eq. (9)) as $|\Phi(t)\rangle=\sum_{p} D_{p}(t) e^{-i E_{p} t}\left|\psi_{p}\right\rangle$, where $D_{p}(t)$ are the time-dependent expansion coefficients and $E_{p}$ are the excitonic energies of the corresponding eigenstates. Substitution of this wavefunction into the TDSE with the Hamiltonian (1) leads eventually to the set of coupled differential equations:

$$
\dot{D}_{q}(t)=i \varepsilon_{0}(t) \cdot \sum_{p} D_{p}(t) e^{-i\left(E_{p}-E_{q}\right) t}\left(\mathbf{M}_{q p}^{+} e^{-i \omega t}+\mathbf{M}_{q p}^{-} e^{i \omega t}\right)
$$

with the excitonic eigenstates $q$ and $p$ coupled by the electric field. The following matrix elements $\mathbf{M}_{q p}^{ \pm}$play the role of transition dipole moments for the extended nanostructure:

$$
\mathbf{M}_{q p}^{ \pm}=\sum_{I} e^{ \pm i \mathbf{k}_{\omega} \cdot \mathbf{R}_{I}} \sum_{i j \ldots z} \sum_{k_{I}^{\prime}} C_{i j \ldots k_{I}^{\prime} \ldots z}^{* q} C_{i j \ldots k_{I} \ldots z}^{p} \boldsymbol{\mu}_{k_{I}^{\prime} k_{I}}^{I}
$$

where $\boldsymbol{\mu}_{k_{I}^{\prime} k_{I}}^{I}$ are the transition dipole moments between states $k_{I}$ and $k_{I}^{\prime}$ of the cluster $I$. These dipole moments can be naturally calculated using the transition charges as

$$
\boldsymbol{\mu}_{k_{I}^{\prime} k_{I}}^{I}=\sum_{a} q_{k_{I}^{\prime} k_{I}}^{I, a} \mathbf{r}_{I, a}
$$

In order to extract information about each individual cluster response from the array wavefunction we employ the reduced density matrix formalism. Calculation of the partial trace of the full array density operator $\mathrm{P}=|\Phi(t)\rangle\langle\Phi(t)|$ over all clusters except the one of interest yields the reduced density operator for that cluster

$$
\mathrm{P}_{I}=\sum_{p q} D_{p}(t) D_{q}^{*}(t) e^{-i\left(E_{p}-E_{q}\right) t} \sum_{i j \ldots z} \sum_{k_{I}^{\prime}} C_{i j \ldots k_{I}^{\prime} \ldots z}^{* q} C_{i j \ldots k_{I} \ldots z}^{p}\left|\Psi_{k_{I}}^{I}\right\rangle\left\langle\Psi_{k_{I}^{\prime}}^{I}\right| .
$$

The diagonal elements of the reduced density matrix in the basis $\left|\Psi_{k_{I}}^{I}\right\rangle$ provide the population of the corresponding electronic states of the selected cluster $I$, and the expectation value of any observable of interest can be determined as a trace of the product of the corresponding operator with the reduced density operator.

In the current study we aim to simulate the spatial distribution of the electric field produced by an array constructed of nanosized subunits with the distance between them smaller than the size of an individual component. For this purpose we represent individual constituents of the array by sets of point charges located at the nuclear positions with the time-dependent charge determined as follows: 


$$
q_{I, a}(t)=\operatorname{Tr}\left(\mathrm{P}_{I} \mathrm{q}_{I, a}\right) .
$$

Under this assumption, the electric field produced by each single point charge can be calculated using the classical electromagnetic-field expression [54] and the total electric field distribution is obtained by superposition of all point charge contributions:

$$
\mathbf{E}(\mathbf{r}, t)=\sum_{I}^{N} \sum_{a} \frac{q_{I, a}(t)\left(\mathbf{r}-\mathbf{r}_{I, a}\right)}{\left|\mathbf{r}-\mathbf{r}_{I, a}\right|^{3}}
$$

E. Approximate iterative approach for the simulation of electromagnetic field propagation

The transition-charge-based quantum mechanical approach described above allows for the simulation of electron dynamics in any system constructed of well-distin-guishable subunits. In practice, this approach faces the problem of exponential growth of the Hamiltonian matrix with increasing number of subunits. Indeed, the size of the basis (5) grows as the number of states per cluster to the power of $N$, with $N$ being the number of clusters. On the other hand, it is mandatory to include tens of electronic excited states per cluster in order to describe real systems and take into account possible nonlinear effects $[10,55]$. Therefore, in order to be able to treat large systems we modify the approximate iterative procedure presented in [44] to include the transition-charge-based interaction between clusters.

Here we briefly outline the main steps of the iterative procedure. In general, we solve the following TDSE for each single subunit

$$
i \frac{\partial}{\partial t}\left|\Phi_{I}(t)\right\rangle=\left(\mathrm{H}_{I}^{0}+\sum_{\substack{J=1 \\ J \neq I}}^{N} \mathrm{~V}_{I J}+\mathrm{V}_{I}^{e x t}\right)\left|\Phi_{I}(t)\right\rangle,
$$

with the single-cluster operators $\mathrm{H}_{I}^{0}$ and $\mathrm{V}_{I}^{e x t}$ the same as described in Sec. II A. The cluster-cluster interaction operator $\mathrm{V}_{I J}$ we approximate with

$$
\mathrm{V}_{I J} \approx \sum_{a b} \frac{\mathrm{q}_{I, a} q_{J, b}(t)}{\left|\mathbf{r}_{I, a}-\mathbf{r}_{J, b}\right|} .
$$

Here instead of the operator $\mathrm{q}_{J, b}$ we use its expectation value determined according to Eq. (22) but with the density operator equal to $\left|\Phi_{J}(t)\right\rangle\left\langle\Phi_{J}(t)\right|$. This means that in order to 
determine the wavefunction $\left|\Phi_{I}(t)\right\rangle$ the wavefunctions $\left|\Phi_{J}(t)\right\rangle$ of all other clusters in the array are required, which is impossible without knowledge of $\left|\Phi_{I}(t)\right\rangle$. Therefore the Eqs. (24)-(25) are solved iteratively with the initial guess for time-dependent charges equal to ground-state partial charges of the corresponding atoms. The deviation between the charges obtained in subsequent iterations is taken as a convergence criterion:

$$
\delta=\frac{1}{T} \sum_{I} \sum_{a} \int_{0}^{T}\left|q_{I, a}^{i+1}(t)-q_{I, a}^{i}(t)\right| d t<\epsilon,
$$

where $T$ is the simulation time. Finally, the electric field is calculated according to Eq. (23) with the time-dependent charges obtained in the last iteration.

\section{RESULTS AND DISCUSSION}

\section{A. Optical and electronic properties of PorphAg $\mathrm{Amonomer}_{4}$}

In order to carry out simulations of electron dynamics and electric field propagation in

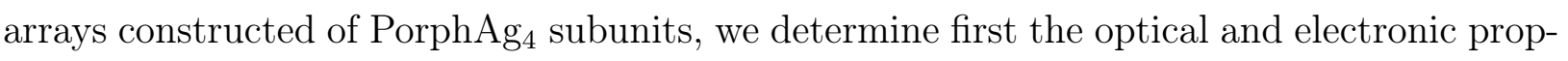

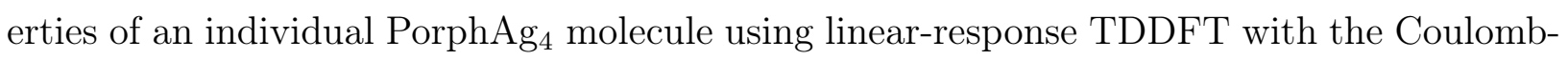
attenuated B3-LYP functional [56] and the triple zeta valence plus polarization atomic Gaussian basis set (TZVPP) [57]. For silver, the atomic basis set and relativistic 11 electron effective core potential optimized to describe the excited electronic states of silver clusters $[3,58,59]$ was used. The minimal energy structure of the PorphAg $\mathrm{Ag}_{4}$ molecule is presented in Fig. 1. For this nuclear configuration the 34 lowest electronic excited states were calculated at the same level of theory and the one-electron and transition densities between all these states were determined according to Eqs. (15)-(17). Subsequently, based on the electrostatic potential fit (2) the set of corresponding partial charges and transition charges was obtained. In order to reduce the number of point charges, those located on hydrogen atoms were set to zero prior to the fitting procedure.

The analysis reveals [48] that the Porph $\mathrm{Ag}_{4}$ monomer can be considered as a positively charged $\mathrm{Ag}_{3}^{+}$cluster placed on a negatively charged PorphAg- support, making the whole system electrically neutral. Moreover, it can be shown [48] that the lowest bright optical transitions of the $\mathrm{Ag}_{3}^{+}$cluster are almost fully preserved in the PorphAg 4 molecule. The absorption spectrum of the bare $\mathrm{Ag}_{3}^{+}$cluster calculated at the CAM-B3-LYP/TZVPP level of 


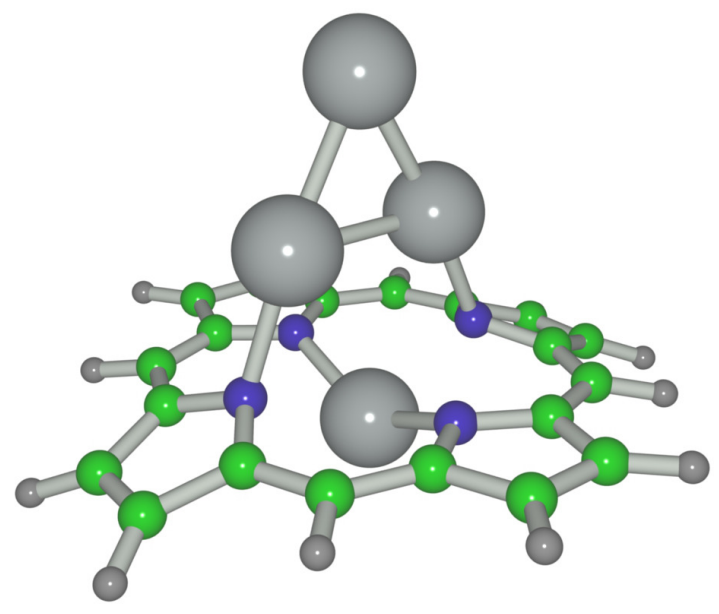

Figure 1: The minimal energy structure of PorphAg 4 calculated at the CAM-B3LYP/TZVPP level of theory.

theory is presented in Fig. 2 (a). The lowest absorption peak of $\mathrm{Ag}_{3}^{+}$contains transitions to the two degenerate electronic excited states $S_{0} \rightarrow S_{1}$ and $S_{0} \rightarrow S_{2}$ with the transition dipole moments lying in the plane of the cluster (directions $\mathrm{Y}$ and Z in Fig. 2 (a)) and having the Slike to P-like excitation character. The second absorption peak is due to $S_{0} \rightarrow S_{3}$ excitation with the transition dipole moment perpendicular to the plane of the cluster (direction $\mathrm{X}$ ). The corresponding transition densities, which in the current realisation are real quantities, are shown in Fig. 2 (b).

In the PorphAg ${ }_{4}$ molecule excitations with the transition density localized mainly on the $\mathrm{Ag}_{3}^{+}$part can be found as well (cf. Fig. 2 (c)-(d)). These are $S_{0} \rightarrow S_{13}(\mathrm{Y}), S_{0} \rightarrow S_{15}(\mathrm{Z})$, and $S_{0} \rightarrow S_{31}(\mathrm{X})$ electronic state with a dominant $\mathrm{S} \rightarrow \mathrm{P}$ excitation character localized on the cluster subunit. Due to the interaction with the PorphAg ${ }^{-}$support, the degeneracy of the Y and $\mathrm{Z}$ transitions is broken and the $\mathrm{X}$ transition is shifted to lower energies. The amount of the transition density localized on the PorphAg ${ }^{-}$substrate for these excitations is relatively small, indicating that the transitions characteristic to the pure $\mathrm{Ag}_{3}^{+}$cluster are preserved in the PorphAg 4 subunit. The comparison between PorphAg 4 and PorphAg ${ }^{-}$spectra (the latter is shown in Fig. 2 (c) as the shaded area) confirms that the broad absorption band in the PorphAg 4 spectrum at 4.0-4.5 eV is formed not only by the transitions localized 


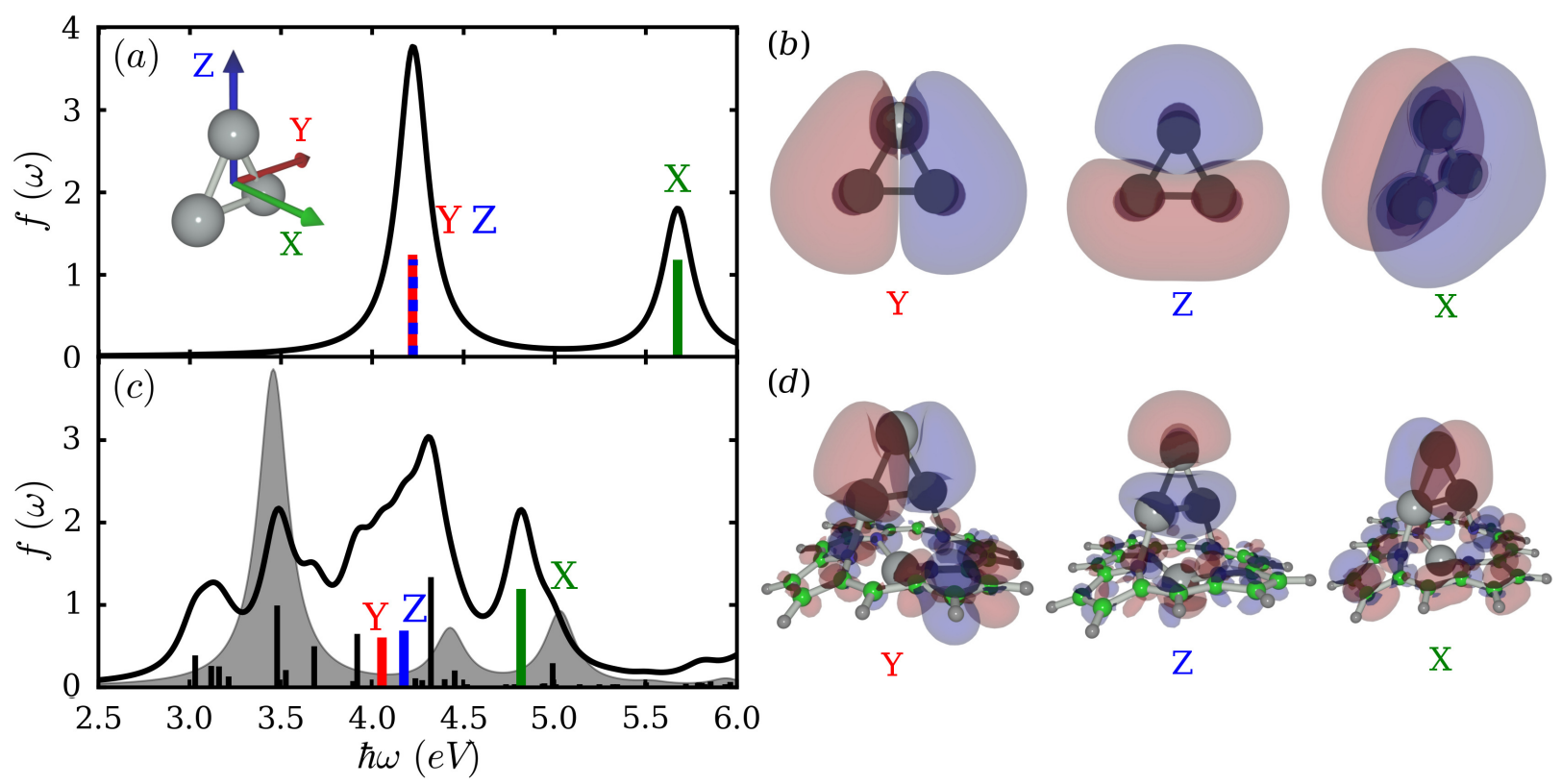

Figure 2: (a) Absorption spectrum of $\mathrm{Ag}_{3}^{+}$cluster. The nuclear configuration of the cluster together with the directions of the selected transition dipole moments are shown in the inset; (b) transition densities of the optical transitions highlighted in (a) calculated using Eq. (16). The positive lobe is denoted with red and the negative one with blue colors; (c) absorption spectrum of the PorphAg 4 molecule. The absorption spectrum of the substrate $\mathrm{PorphAg}^{-}$molecule alone is presented as the shaded area in the background; (d) transition densities of the optical transitions marked in (c).

mainly on the $\mathrm{Ag}_{3}^{+}$cluster, but also by the hybrid ones involving cluster and substrate simultaneously. Notably, the pure PorphAg- molecule possesses no significant absorption in this region. The further detailed analysis of the optical properties of the $\mathrm{Ag}_{3}^{+}$cluster vs. $\mathrm{PorphAg}_{4}$ molecule is presented elsewhere [48].

\section{B. Light-induced exciton dynamics dynamics in $\mathbf{A g}_{3}^{+}$cluster and PorphAg $\mathrm{A}_{4}$ dimers}

We first apply our methodology to study the electron dynamics in the PorphAg $\operatorname{dimer}_{4}$ and compare it to the pure $\mathrm{Ag}_{3}^{+}$cluster dimer in order to validate the approximate iterative approach presented in Section II E versus the full quantum approach from Section II D .

The laser-driven electron dynamics in the dimers was induced by an external short laser pulse with the temporal profile described by a Gaussian function 


$$
\varepsilon_{0}(t)=\varepsilon_{\max } \exp \left(-\frac{\left(t-t_{0}\right)^{2}}{2 \sigma^{2}}\right),
$$

with the full width at half maximum (FWHM) of $19 \mathrm{fs}(\sigma=8.0 \mathrm{fs})$, the peak pulse strength $\varepsilon_{\max }=5 \cdot 10^{-3} E_{\mathrm{h}} / e a_{0}$, and the pulse center at $t_{0}=20 \mathrm{fs}$. The central frequency of the pulse was chosen to be resonant with the lowest intense transition localized on the silver cluster (denoted with $\mathrm{Y}$ in Fig. 2), which for the bare $\mathrm{Ag}_{3}$ cluster is equal to $4.22 \mathrm{eV}$ and for the $\mathrm{PorphAg}_{4}$ to $4.05 \mathrm{eV}$. The field propagates along the line connecting the subunits' centers and is polarized in the plane containing the porphyrin layer (see inset in Fig. 3 (c)). For the $\mathrm{Ag}_{3}^{+}$dimer the orientation of the clusters and the electric field is the same. In both cases the distance between the cluster centers in the dimers is equal to $9 \AA$.

The population dynamics of the first $\mathrm{Ag}_{3}^{+}$cluster in the dimer is presented in Fig. 3 (a). The population of the second cluster is almost the same and is not presented here. The simulation was performed using the full-quantum approach based on the dipole-dipole intercluster coupling as described in [44]. The distance between the clusters is large enough compared to the single cluster size, making it possible to use the dipole approximation in such systems. It is seen that under the action of the pulse the electronic population is first transferred to the $S_{1}$ electronic excited state, which is resonant to the laser field frequency. Subsequently, part of the electronic population is transferred to the $S_{2}$ state, although the transition dipole moment to the $S_{2}$ state is orthogonal to the laser field polarization. Notably, when a single cluster is irradiated by the same laser pulse, no population transfer to the $S_{2}$ state is observed, which suggests that the effect is purely due to the intercluster coupling.

Indeed, since the single cluster is charged, the matrix elements (7) and (8) between the basis functions $\left|\phi_{01}\right\rangle,\left|\phi_{02}\right\rangle,\left|\phi_{10}\right\rangle$, and $\left|\phi_{20}\right\rangle$ are nonzero due to the charge-dipole interaction. Because the $S_{1}$ and $S_{2}$ states are energetically degenerate, these four basis functions mix to form the four lowest excitonic eigenstates of the array Hamiltonian

$$
\begin{aligned}
& \left|\psi_{1,4}\right\rangle=0.47\left(\left|\phi_{01}\right\rangle \pm\left|\phi_{10}\right\rangle\right) \mp 0.53\left(\left|\phi_{02}\right\rangle \mp\left|\phi_{20}\right\rangle\right), \\
& \left|\psi_{2,3}\right\rangle=0.53\left(\left|\phi_{01}\right\rangle \mp\left|\phi_{10}\right\rangle\right) \mp 0.47\left(\left|\phi_{02}\right\rangle \pm\left|\phi_{20}\right\rangle\right) .
\end{aligned}
$$

The electronic excitation energies of these four states lie in the range of 4.16-4.30 eV and are resonant to the laser pulse energy. Since both $\left|\psi_{1}\right\rangle$ and $\left|\psi_{3}\right\rangle$ eigenstates are symmetric with 
respect to $\left|\phi_{01}\right\rangle$ and $\left|\phi_{10}\right\rangle$ and antisymmetric with respect to $\left|\phi_{02}\right\rangle$ and $\left|\phi_{20}\right\rangle$, the transition dipole moments (19) from the dimer ground state to these eigenstates are parallel to $\boldsymbol{\mu}_{01}$ in agreement with Eq. (19) and thus to the direction of the laser pulse polarization. Therefore, the electron population of the dimer is first transferred to these two states. Subsequently, another group of dimer eigenstates is populated, namely, the states $\left|\psi_{7,9,10}\right\rangle$, which contain significant contributions from the $\left|\phi_{11}\right\rangle,\left|\phi_{12}\right\rangle,\left|\phi_{21}\right\rangle$, and $\left|\phi_{22}\right\rangle$ basis functions. The excitation energies of these states are approximately twice the energy of the exciting laser pulse and the transition dipole moments from the $\left|\psi_{1}\right\rangle$ or $\left|\psi_{3}\right\rangle$ states are parallel to the laser polarization vector. In this manner, the electron population oscillates between $\left|\psi_{0}\right\rangle,\left|\psi_{1,3}\right\rangle$, and $\left|\psi_{7,9,10}\right\rangle$ states until the laser pulse ceases.

In order to retrieve the electron population dynamics in each single cluster, the diagonal elements of the reduced density matrix (21) are calculated. Since the contributions of the $S_{1}$ and $S_{2}$ single-cluster states to the involved dimer eigenstates are almost equal in magnitude, the phase of the eigencoefficients $C_{i j}^{p}$ (see Eq. (10)) and time-dependent expansion coefficients $D_{p}(t) e^{-i E_{p} t}$ determines the population of the single-cluster states. When the laser field starts to act, the phase shift between $D_{1}(t) e^{-i E_{1} t}$ and $D_{3}(t) e^{-i E_{3} t}$ is small and the relative phase between $C_{i j}^{1}$ and $C_{i j}^{3}$ plays a decisive role. Since both $\left|\psi_{1}\right\rangle$ and $\left|\psi_{3}\right\rangle$ eigenstates are symmetric with respect to $\left|\phi_{01}\right\rangle$ and $\left|\phi_{10}\right\rangle$ and antisymmetric with respect to $\left|\phi_{02}\right\rangle$ and $\left|\phi_{20}\right\rangle$, the interference between these eigenstates leads to electron population transfer to the $S_{1}$ state only in the single-cluster picture. The increase of the phase shift between the time-dependent coefficients as well as the change of their magnitude leads to subsequent electron population oscillations between the $S_{1}$ and $S_{2}$ single-cluster degenerate states even after the laser pulse ceases (after $\sim 40$ fs of simulation time).

When the substrate is taken into account, the electronic population dynamics changes significantly (see Fig. $3(\mathrm{~b})$ ). The electronic excited states $S_{13}$ and $S_{15}$ which correspond to the $S_{1}$ and $S_{2}$ states of the bare cluster, are not degenerate and thus almost no population transfer to the $S_{15}$ state is observed. The effect of charge-dipole interaction, which played an important role for $\mathrm{Ag}_{3}^{+}$dimer, is now compensated by interaction with the negative charge localized on porphyrin. Instead, during the external laser pulse action some part of the electronic population is transferred to the $S_{19}$ state, which has the transition density almost equally distributed between the $\mathrm{Ag}_{3}^{+}$part and porphyrin. After the pulse ceases, the population of this state vanishes and the population of the excited $S_{13}$ and ground $S_{0}$ 


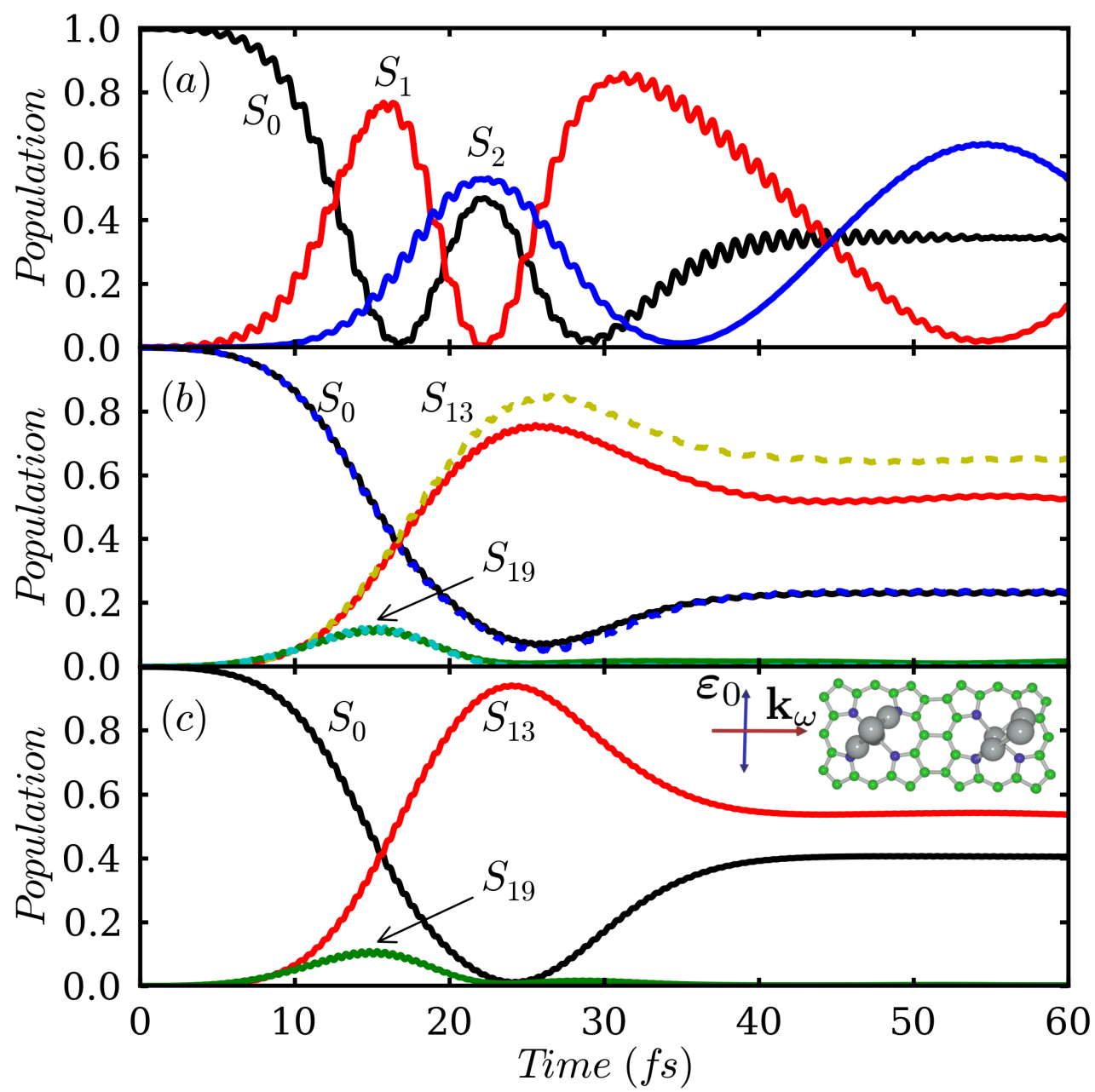

Figure 3: (a) Electronic population dynamics in the first cluster of the $\mathrm{Ag}_{3}^{+}$dimer induced by the external laser pulse; (b) electron population dynamics in the first subunit of the Porph $\mathrm{Ag}_{4}$ dimer. The simulations were performed using the full-quantum method described in Sec. IID (solid lines) and the approximate iterative approach presented in Sec. IIE (dashed lines); (c) population dynamics in the first subunit of the $\mathrm{PorphAg}_{4}$ dimer calculated using the full-quantum approach based on the dipole interaction between clusters as presented in Ref. [44]. The structure of the dimer considered in (b) and (c) as well as the external laser field configuration are shown in the inset.

electronic states remains constant with time. The results obtained using the approximate iterative approach presented in Sec. II E (see Fig. 3 (b), dashed lines) reproduce the fullquantum calculations (Fig. 3 (b), solid lines) quite accurately.

For comparison, we simulated the electronic population dynamics in the PorphAg $\mathrm{Aimer}_{4}$ 
using the full-quantum approach presented in [44], in which the clusters within the array interact between each other as point dipoles. The results are shown in Fig. 3 (c). It is evident that the latter method overestimates the excited state population during the laser pulse action as well as the final ground state population. However, the main features of the population dynamics are preserved, which is mainly due to the fact that the transition densities to the excited states involved in the dynamics are localized on the $\mathrm{Ag}_{3}^{+}$part and the distance between them in the dimer is large enough.

The essential quantities needed to simulate the electric field distribution in the PorphAg 4 array are the time-dependent partial charges (Eq. (22)). We compare the charges located on the silver atoms A, B, and C (see scheme in Fig. 4 (a)) calculated using the fullquantum approach (Sec. II D) and the iterative approach (IIE). The convergence of the iterative method was reached after $\sim 20$ iterations, the relative difference between the timedependent charges obtained in each two subsequent iterations calculated according to Eq. (26) is plotted in Fig. 4 (b). The time-dependent charges are presented in Fig. 4 (c) and (d). The charges on atoms A and B strongly oscillate in the counter-phase, while the charge on C remains almost unchanged during the electron dynamics. It is evident that the iterative approach allows for simulation of such time-dependent observables with good accuracy. The average difference between the two methods is about $4 \%$.

\section{Exciton dynamics in a PorphAg 4 -ring}

Finally we apply the developed iterative approach to the simulation of light-exciton dynamics in a ring constructed of $10 \mathrm{PorphAg}_{4}$ clusters shown in Fig. 5. The ring is irradiated by a circularly polarized light with the wave vector perpendicular to the ring plane and the polarization vector rotating in the plane of the ring. Other parameters of the laser field are the same as stated in Sec. III B. Since the $\mathrm{Ag}_{3}^{+}$cluster is oriented along the diagonal of the square representing the porphyrin molecule, the ring formed in this manner is asymmetric with respect to the clockwise and anti-clockwise rotations. Therefore different electron population dynamics induced by two different directions of the polarization plane rotation can be expected.

The population dynamics of one of the subunits in the ring under the action of either an external right-handed or left-handed polarized laser pulse are presented in Fig. 6. The other 
subunits demonstrate essentially the same dynamics and are not shown here. It is evident, that the two different directions of the polarization-plane rotation induce different electronic state population dynamics. Under the action of left-handed polarized pulse significant part of the electronic population is transferred to the excited state $S_{10}$, which is not the case for the right-handed polarized light. Additionally, the ground state population after the pulse has ceased is more than two times lower for the left-handed polarized pulse and the population oscillations between the $S_{9}$ and $S_{13}$ excited electronic states are more pronounced in this case. This means, that the dipole-quadrupole and quadrupole-quadrupole interactions between the subunits play an important role for this system.

The different electronic population dynamics results in different spatio-temporal electric field distribution induced by the two circularly polarized pulses in the ring. We use Eq. (23) to evaluate the electric field energy localized around the silver cluster in a PorphAg $\mathrm{Subunit}_{4}$ Since the electric field energy is proportional to the square of the electric field strength, we integrate $\left|E^{2}\right|$ over a sphere of $2.4 \AA$ radius centered at the $\mathrm{Ag}_{3}^{+}$geometrical center. The radius of the sphere was chosen so that the sphere contained all three silver atoms but is well separated from the porphyrin plane. The results are presented in Fig. 7. The elelctric field energy was divided into the quasi-static (shown with solid black line) and fastoscillating dynamic (semi-transparent red line) fractions. The quasi-static part represents the contribution of the slowly-varying time-dependent dipole moment of a subunit to the total electric field, while the dynamic one reflects the instantaneous changes in the electron density. With the solid red line the average value of the fast-oscillating fraction of the electric field energy is shown. It is clearly seen, that while the right-handed polarized laser pulse provides almost equal distribution of the electric field energy between the quasi-static and dynamic components, the left-handed polarized one induces a much higher quasi-static component as compared to the dynamic one.

The observed difference can be interpreted in terms of electronic population dynamics caused by two pulses. Indeed, irradiation with the left-handed polarized laser pulse leads to excitation, among others, of the $S_{9}$ and $S_{10}$ electronic states. These states possess relatively high permanent dipole moments, which contribute to the total time-dependent dipole moment of the subunit proportionally to the actual population of the states. Therefore, the maxima in the $S_{9}$ electronic population correspond to the maxima in the quasi-static component of the electric field energy. In the case of right-handed polarized laser pulse, no 
population transfer to the $S_{10}$ state occurs, thus decreasing the quasi-static contribution to the total electric field. On the other hand, the fast-oscillating component of the electric field arises due to coupling between the ground and $S_{13}$ excited electronic states, because the transition dipole moment between these states is high enough and lies in the plane which the polarization vector of the laser pulse belongs to. Additionally, the excitation energy of the $S_{13}$ state is resonant to the laser photon energy. Consequently, the dynamic part of the electric field energy produced by each subunit oscillates with a frequency close to the driving electric field and with an amplitude reflecting the electronic populations of the ground and $S_{13}$ states. In particular, comparing Figs. 6 and 7 it can be clearly seen, that after the laser pulse has ceased and no more electronic population transfer from and to the ground state occurs, the maxima of the dynamic part of the electric field coincide with the maxima of the $S_{13}$ state population.

As a result of the interplay between the quasi-static and dynamic components, the total energy of the electric field produced by the PorphAg ${ }_{4}$ subunit demonstrates different spatial and temporal behavior. For the left-handed polarized laser pulse the quasi-static part significantly exceeds the dynamic one and the total electric field exhibits pronounced oscillations corresponding to the $S_{9}$ electronic state population maxima. On the other hand, when the $\mathrm{PorphAg}_{4}$ ring is irradiated with the right-handed polarized light, the two components are approximately of the same magnitude. Since these components vary with opposite phases (cf. Fig. 7 top), the oscillations of the total electric field energy are quenched.

Finally, in order to compare the spatial electric field energy distribution, we simulate the electric field distribution in the plane parallel to the ring plane and passing through geometrical centers of the $\mathrm{Ag}_{3}^{+}$clusters. Fig. 8 illustrates the spatial configuration of the electric field energy at different instants of time. It is seen that the two circular light polarizations with different direction of the polarization-plane rotation create highly spatially and temporally inhomogeneous electric field localized at the inner or outer atoms of the silver clusters. Due to this fact, the field strength at the center of the ring is in average lower for the right-handed polarization than for the left-handed one. The bottom panel in Fig. (8) shows the difference between the electric field energies produced by the ring at the current instant of time to the initial electric field distribution in the ground state for the left-handed polarized light. It is seen, that at the beginning ( $2 \mathrm{fs}$ ) the electric field is redistributed between two silver atoms and later additional decrease of the electric field outside the cluster plane is 
observed and the electric field is localized in the plane of the cluster.

\section{CONCLUSION}

In conclusion, we have presented a theoretical approach for the simulation of the light propagation and exciton dynamics in structured arrays of small noble-metal clusters deposited on an organic template support. Within the presented methodology the interaction between subunits is described using the ab initio parameterized transition charge method, which enables one to take into account dipole-quadrupole, quadrupole-quadrupole, and higher terms in the multipole expansion of the Coulomb interaction, making the method applicable to nanostructures with a separation between individual constituents smaller or comparable to their size. The excitonic Hamiltonian used to simulate the quantum temporal evolution of the array under the action of an external electric field is constructed based on first principles TDDFT calculations, thus allowing for a realistic description of the electronic structure of the array including many electronic states per unit. Based on the numerical solution of the TDSE with such a coupled exciton Hamiltonian the set of time-dependent partial charges is determined, which is further used to simulate the spatio-temporal electric field distribution created by the array. The use of the set of time-dependent charges instead of time-dependent dipole moments allows for the investigation of the highly inhomogeneous electric field localized around different parts of same subunit. The developed iterative approach extends the applicability of the method to large arrays consisting of a significant number of constituents with tens of electronic excited states per subunit.

The methodology presented in the current work can be combined with our field-induced surface-hopping (FISH) method [60] to include the nuclear dynamics of the studied aggregates into the simulations. Although in the current work the simulation time period is relatively short for the nuclear rearrangement to have significant impact on the electronic structure of the noble-metal clusters [10,61,62], in further investigations the simulation time scale can be extended to study the effect of laser-induced coupled electron-nuclear dynamics on the light propagation in noble-metal cluster aggregates.

The proposed methodology was used to simulate the optical response of the pure $\mathrm{Ag}_{3}^{+}$ cluster dimer as well as the PorphAg 4 dimer (cluster array deposited on porphyrin support). It was demonstrated that the electronic state population dynamics differs significantly when 
the porphyrin support is included into simulations. Additionally, the latter system was used to validate the approximate iterative approach.

Finally, using the iterative approach the optical response and the spatio-temporal elec-

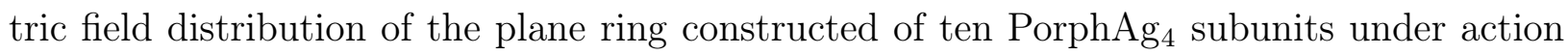
of circularly polarized light was simulated. It was found that in such systems different directions of the polarization-plane rotation lead to different electron population dynamics and thus to different electric field distributions. Such systems are promising building blocks for nanooptical devices switching the regimes of operation under circularly polarized external laser pulses.

\section{Acknowledgments}

The authors are grateful to the Deutsche Forschungsgemeinschaft (DFG) for the financial support via the Priority program "Ultrafast Nanooptics" (SPP 1391) and the ERC Consolidator Grant DYNAMO (Grant No. 646737).

[1] Sanchez, A.; Abbet, S.; Heiz, U.; Schneider, W. D.; Hakkinen, H.; Barnett, R. N.; Landman, U. J. Phys. Chem. A 1999, 103, 9573.

[2] Shipway, A. N.; Katz, E.; Willner, I. Chem. Phys. Chem. 2000, 1, 18-52.

[3] Bonačić-Koutecký, V.; Veyret, V.; Mitrić, R. J. Chem. Phys. 2001, 115, 10450.

[4] Peyser, L. A.; Vinson, A. E.; Bartko, A. P.; Dickson, R. M. Science 2001, 291, 103.

[5] Zheng, J.; Zhang, C. W.; Dickson, R. M. Phys. Rev. Lett. 2004, 93, 077402.

[6] Lal, S.; Link, S.; Halas, N. J. Nat. Photon. 2007, 1, 641-648.

[7] Johnson, G. E.; Mitrić, R.; Bonačić-Koutecký, V.; Castleman, J. A. W. Chem. Phys. Lett. 2009, 475, 1-9.

[8] Benson, O. Nature 2011, 480, 193-199.

[9] Lang, S. M.; Bernhardt, T. M. Phys. Chem. Chem. Phys. 2012, 14, 9255-9269.

[10] Lisinetskaya, P. G.; Mitrić, R. Phys. Rev. A 2011, 83, 033408.

[11] Polyushkin, D. K.; Marton, I.; Racz, P.; Dombi, P.; Hendry, E.; Barnes, W. L. Phys. Rev. B 2014, 89 . 
[12] Seydack, M. Biosens. Bioelectron. 2005, 20, 2454-2469.

[13] Anker, J. N.; Hall, W. P.; Lyandres, O.; Shah, N. C.; Zhao, J.; Van Duyne, R. P. Nature Mater. 2008, 7, 442-453.

[14] Hahn, M. A.; Singh, A. K.; Sharma, P.; Brown, S. C.; Moudgil, B. M. Anal. Bioanal. Chem. 2011, 399, 3-27.

[15] Sailor, M. J.; Park, J.-H. Adv. Mater. 2012, 24, 3779-3802.

[16] Quinten, M.; Leitner, A.; Krenn, J. R.; Aussenegg, F. R. Opt. Lett. 1998, 23, 1331-1333.

[17] Maier, S. A.; Brongersma, M. L.; Kik, P. G.; Meltzer, S.; Requicha, A. A. G.; Atwater, H. A. Adv. Mater. 2001, 13, 1501-1505.

[18] Palacios, E.; Chen, A.; Foley, J.; Gray, S. K.; Welp, U.; Rosenmann, D.; Vlasko-Vlasov, V. K. Adv. Opt. Mater. 2014, 2, 394-399.

[19] Hasobe, T.; Imahori, H.; Kamat, P. V.; Ahn, T. K.; Kim, S. K.; Kim, D.; Fujimoto, A.; Hirakawa, T.; Fukuzumi, S. J. Am. Chem. Soc. 2005, 127, 1216-1228.

[20] Nakayama, K.; Tanabe, K.; Atwater, H. A. Appl. Phys. Lett. 2008, 93.

[21] Ma, H.; Gao, F.; Liang, W. Z. J. Phys. Chem. C 2012, 116, 1755-1763.

[22] Stockman, M. I.; Faleev, S. V.; Bergman, D. J. Phys. Rev. Lett. 2002, 88, 067402.

[23] Brixner, T.; García de Abajo, F. J.; Schneider, J.; Pfeiffer, W. Phys. Rev. Lett. 2005, 95, 093901.

[24] Aeschlimann, M.; Bauer, M.; Bayer, D.; Brixner, T.; Cunovic, S.; Dimler, F.; Fischer, A.; Pfeiffer, W.; Rohmer, M.; Schneider, C.; Steeb, F.; Strueber, C.; Voronine, D. V. Proc. Natl. Acad. Sci. U.S.A. 2010, 10\%, 5329-5333.

[25] Sukharev, M.; Seideman, T. Nano Letters 2006, 6, 715-719.

[26] Aeschlimann, M.; Bauer, M.; Bayer, D.; Brixner, T.; Garcia de Abajo, F. J.; Pfeiffer, W.; Rohmer, M.; Spindler, C.; Steeb, F. Nature 2007, 446, 301-304.

[27] Sukharev, M.; Seideman, T. J. Phys. B 2007, 40, S283-S298.

[28] Tuchscherer, P.; Rewitz, C.; Voronine, D. V.; Javier Garcia de Abajo, F.; Pfeiffer, W.; Brixner, T. Opt. Express 2009, 17, 14235-14259.

[29] Wang, H.; Zou, S. Phys. Chem. Chem. Phys. 2009, 11, 5871-5875.

[30] Rockstuhl, C.; Menzel, C.; Muehlig, S.; Petschulat, J.; Helgert, C.; Etrich, C.; Chipouline, A.; Pertsch, T.; Lederer, F. Phys. Rev. B 2011, 83, 245119.

[31] Willingham, B.; Link, S. Opt. Express 2011, 19, 6450-6461. 
[32] Solis, D.; Willingham, B.; Nauert, S. L.; Slaughter, L. S.; Olson, J.; Swanglap, P.; Paul, A.; Chang, W.-S.; Link, S. Nano Letters 2012, 12, 1349-1353.

[33] Maier, S. A.; Kik, P. G.; Atwater, H. A. Phys. Rev. B 2003, 67, 205402.

[34] Gray, S.; Kupka, T. Phys. Rev. B 2003, 68.

[35] Mitrić, R.; Hartmann, M.; Stanca, B.; Bonačić-Koutecký, V.; Fantucci, P. J. Phys. Chem. A 2001, 105, 8892 .

[36] Bonačić-Koutecký, V.; Burda, J.; Ge, M.; Mitrić, R.; Zampella, G.; Fantucci, R. J. Chem. Phys. 2002, 117, 3120.

[37] Huang, W.; Pal, R.; Wang, L. M.; Zeng, X. C.; Wang, L. S. J. Chem. Phys. 2010, 132, 054305.

[38] Morton, S. M.; Silverstein, D. W.; Jensen, L. Chem. Rev. 2011, 111, 3962-3994.

[39] Stanzel, J.; Neeb, M.; Eberhardt, W.; Lisinetskaya, P. G.; Petersen, J.; Mitrić, R. Phys. Rev. A 2012, 85, 013201.

[40] Ziolkowski, R.; Arnold, J.; Gogny, D. Phys. Rev. A 1995, 52, 3082-3094.

[41] Fratalocchi, A.; Conti, C.; Ruocco, G. Phys. Rev. A 2008, 78.

[42] Lopata, K.; Neuhauser, D. J. Chem. Phys. 2009, 131, 014701.

[43] Sukharev, M.; Nitzan, A. Phys. Rev. A 2011, 84, 043802.

[44] Lisinetskaya, P. G.; Mitrić, R. Phys. Rev. B 2014, 89, 035433.

[45] Lisinetskaya, P. G.; Mitrić, R. Phys. Rev. B 2015, 91.

[46] Krueger, B. P.; Scholes, G. D.; Fleming, G. R. J. Phys. Chem. B 1998, 102, 5378-5386.

[47] Madjet, M. E.; Abdurahman, A.; Renger, T. J. Phys. Chem. B 2006, 110, 17268-17281.

[48] Röhr, M. I. S.; Lisinetskaya, P. G.; Mitrić, R. in preparation 2015,

[49] Tsuda, A.; Furuta, H.; Osuka, A. J. Am. Chem. Soc. 2001, 123, 10304-10321.

[50] Aratani, N.; Kim, D.; Osuka, A. Acc. Chem. Res. 2009, 42, 1922-1934.

[51] Werner, U.; Mitrić, R.; Bonačić-Koutecký, V. J. Chem. Phys. 2010, 132, 174301.

[52] Casida, M. E. In Recent Advances in Density Functional Methods; Chong, D. P., Ed.; World Scientific, Singapore, 1995; p 155.

[53] Dreuw, A.; Head-Gordon, M. Chem. Rev. 2005, 105, 4009.

[54] Feynman, R. P.; Leighton, R. B.; Sands, M. L. Mainly electromagnetism and matter; The Feynman Lectures on Physics; Addison-Wesley, 1963.

[55] Röhr, M. I. S.; Petersen, J.; Wohlgemuth, M.; Bonačić-Koutecký, V.; Mitrić, R. ChemPhysChem 2013, 14, 1377-1386. 
[56] Yanai, T.; Tew, D.; Handy, N. Chem. Phys. Lett. 2004, 393, 51-57.

[57] Schäfer, A.; Huber, C.; R. Ahlrichs, R. J. Chem. Phys. 1994, 100, 5829.

[58] Bonačić-Koutecký, V.; Pittner, J.; Boiron, M.; Fantucci, P. J. Chem. Phys. 1999, 110, 3876.

[59] Sieber, C.; Buttet, J.; Harbich, W.; Félix, C.; Mitrić, R.; Bonačić-Koutecký, V. Phys. Rev. A 2004, 70, 041201.

[60] Mitrić, R.; Petersen, J.; Bonačić-Koutecký, V. Phys. Rev. A 2009, 79.

[61] Mitrić, R.; Petersen, J.; Wohlgemuth, M.; Werner, U.; Bonačić-Koutecký, V.; Wöste, L.; Jortner, J. J. Phys. Chem. A 2011, 115, 3755-3765.

[62] Mitrić, R.; Petersen, J.; Wohlgemuth, M.; Werner, U.; Bonačić-Koutecký, V. Phys. Chem. Chem. Phys. 2011, 13, 8690-8696. 

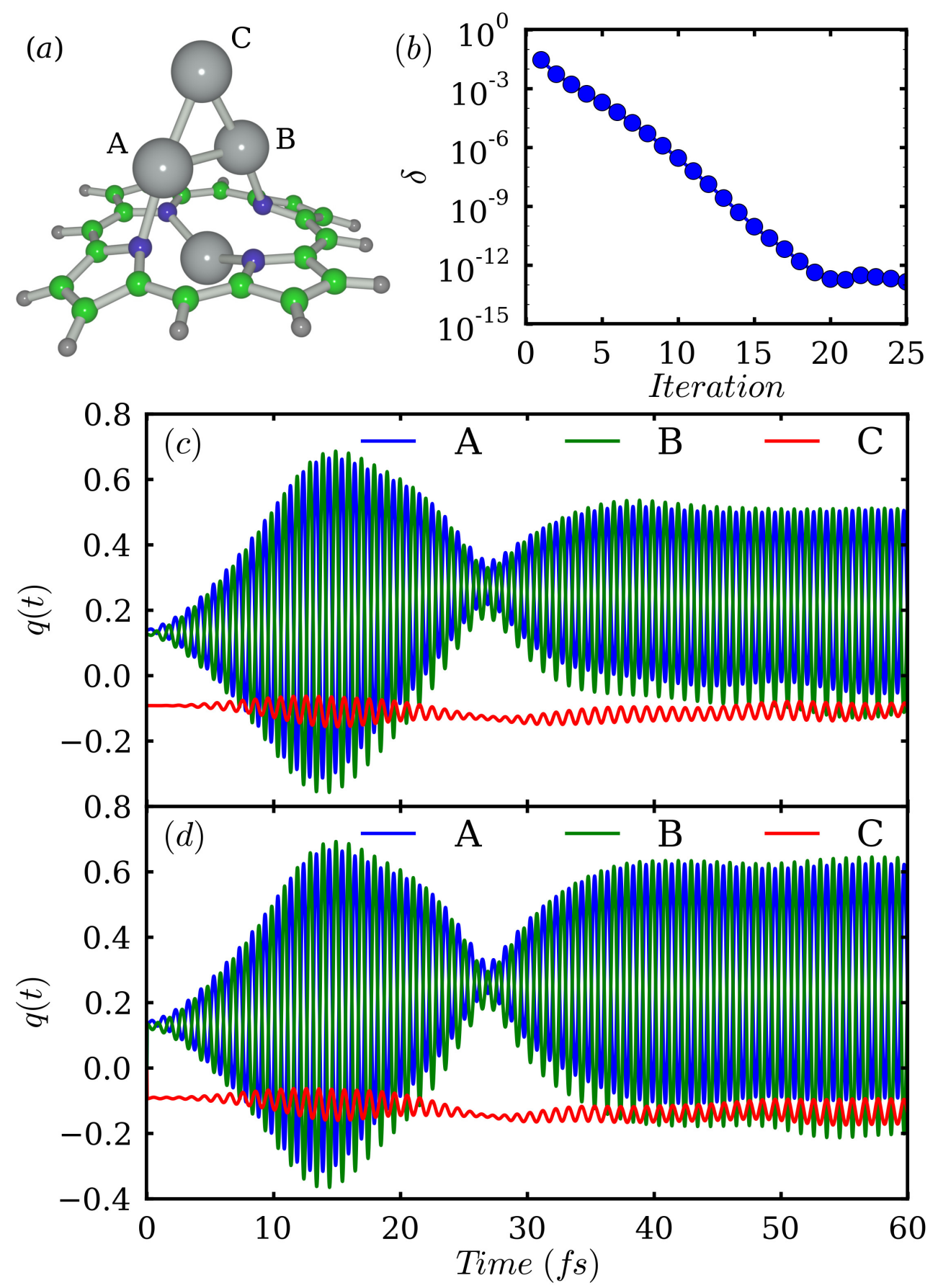

Figure 4: (a) Nuclear configuration of the PorphAg 4 subunit; (b) deviation between the timedependent charges obtained in the given iteration and in the previous one (see Eq. 26); (c) time-dependent charges located on atoms denoted in (a) with A, B, C, obtained in full-quantum simulations; (d) same as (c) but obtained using the iterative approach. 


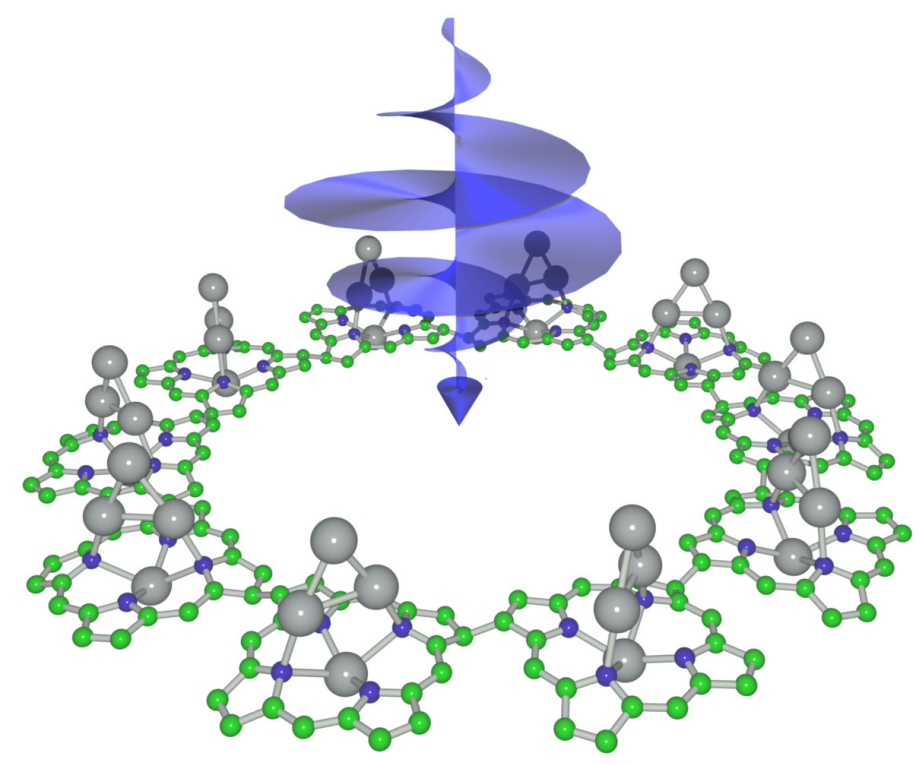

Figure 5: Schematic representation of a ring constructed of ten PorphAg 4 subunits irradiated by a circularly polarized laser pulse.

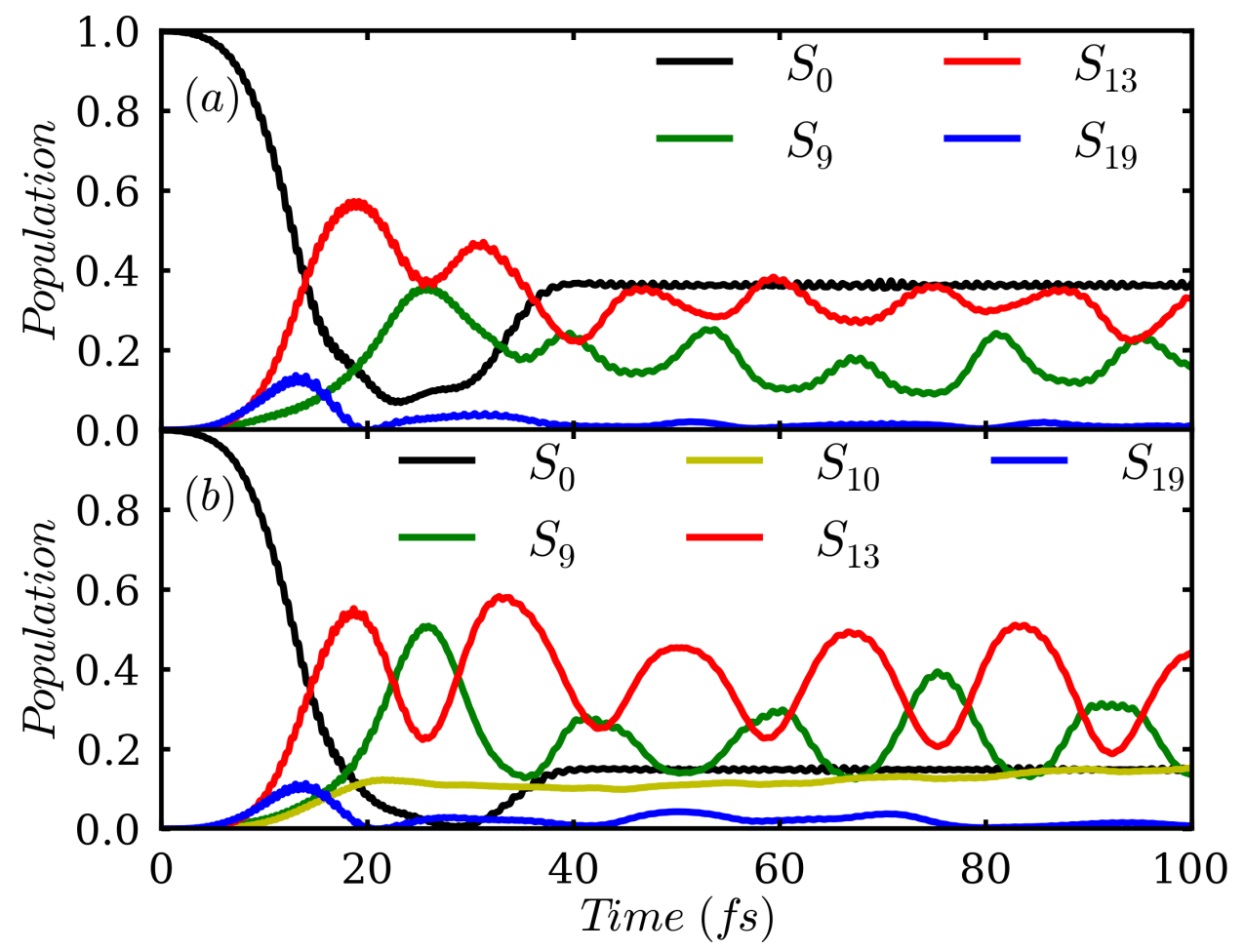

Figure 6: Electron population dynamics induced in the PorphAg 4 ring by (a) right-handed and (b) left-handed polarized laser pulse. 


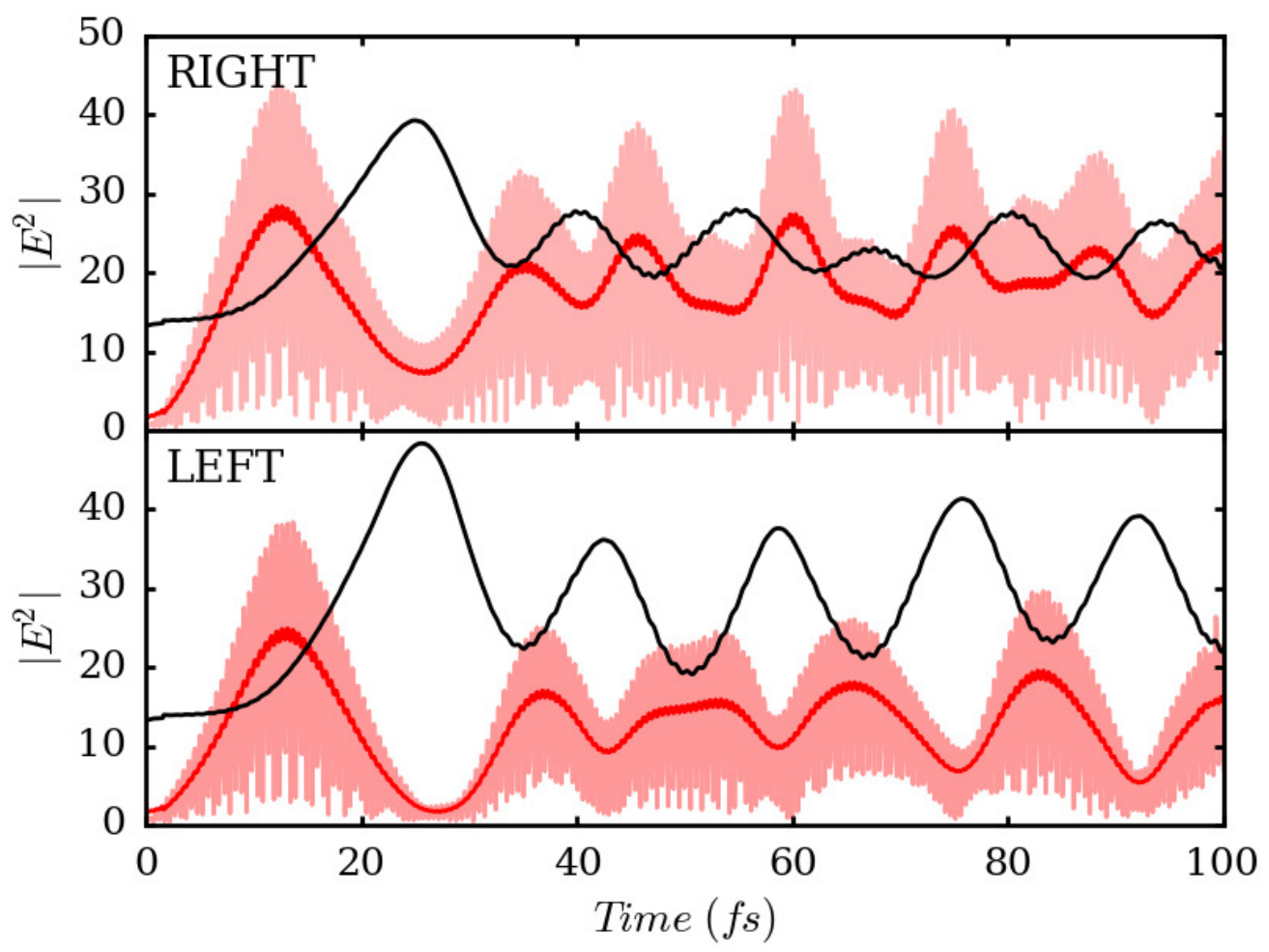

Figure 7: Electric field energy localized around an $\mathrm{Ag}_{3}^{+}$cluster after irradiation with (top) a rightand (bottom) a left-handed polarized laser pulse. 

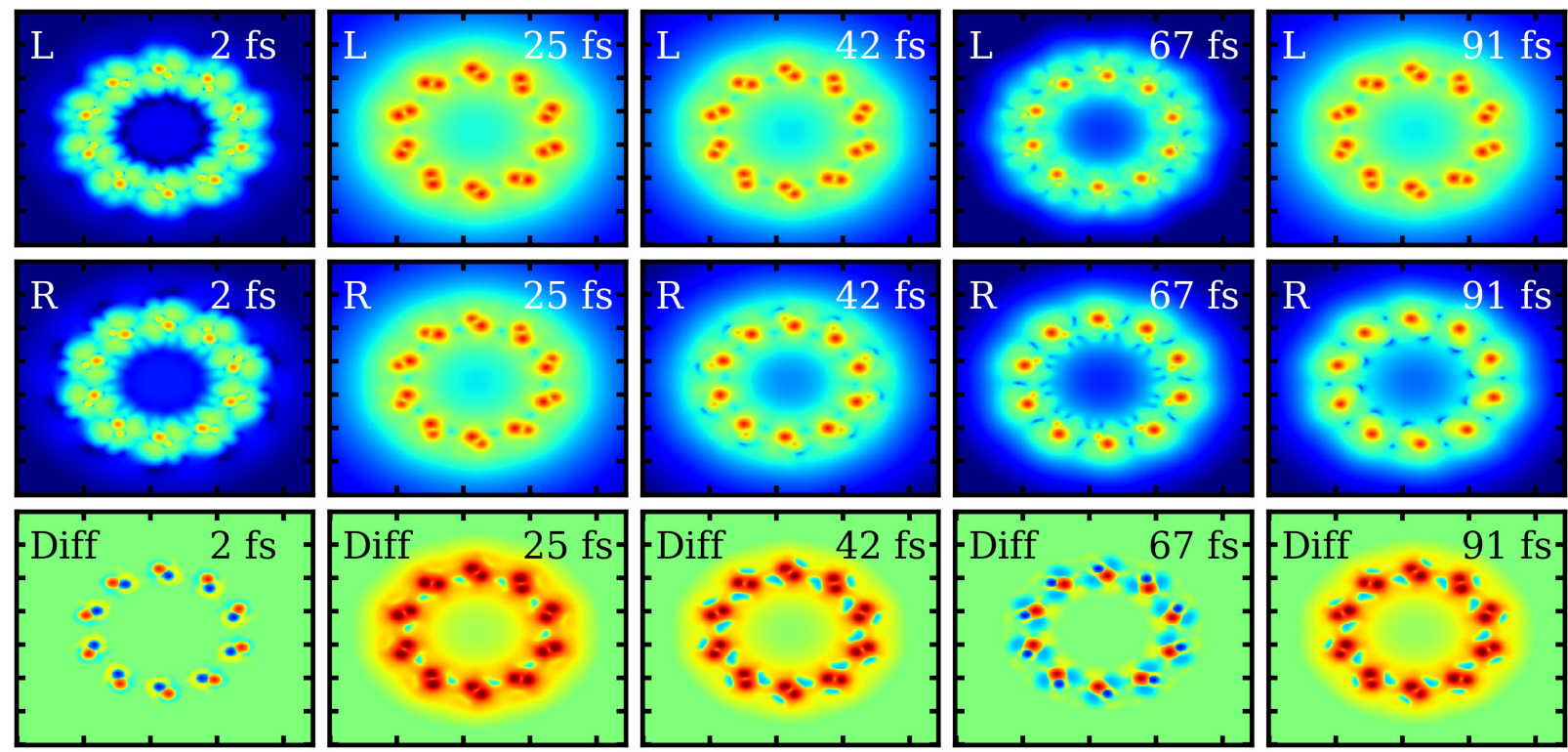

Figure 8: Spatial distribution of electric field energy induced by left- (upper panel, L) and righthanded (middle panel, R) circularly polarized light. The snapshots are taken at selected instants of time specified in each subplot. The magnitude of the electric field energy at each point is shown with the color code from minimal (dark blue) to maximal (red). The lower panel shows the electric field energy difference to the initial electric field distribution for the left-handed polarization (Diff), with blue color indicating decrease, red one increase, and green one indicating zero change. 